\title{
Diagnostic Performance of a New Multifunctional Electrocardiograph During Uninterrupted Chest Compressions in Cardiac Arrest Patients
}

\author{
Genji Shimpuku, MD; Naoto Morimura, MD, PhD; Tetsuya Sakamoto, MD, PhD; \\ Takaaki Isshiki, MD, PhD; Shinya Nagata, PhD; Takahisa Goto, MD, PhD
}

\begin{abstract}
Background: External chest compression is considered to play a significant role in cardiopulmonary resuscitation (CPR), but during a rhythm check, chest compressions must be discontinued to avoid artifacts. A new multifunctional electrocardiograph (ECG; Radarcirc ${ }^{\mathrm{TM}}$ ) has been developed for use in clinical settings.
\end{abstract}

\begin{abstract}
Methods and Results: The performance of the Radarcirc ${ }^{\mathrm{TM}}$ and conventional ECG (CoECG) during CPR was compared in a single-center, non-randomized, sequential self-controlled study. CPR was performed on 41 out-ofhospital cardiac arrest patients. Cardiac rhythm with and without chest compressions during a rhythm check was measured using leads I and II. When the rhythm changed during CPR, it was measured as another waveform. Fifty ECG recordings were obtained, of which 27 were asystole, 18 pulseless electrical activity, and 5 ventricular fibrillation (VF). The area under the receiver-operating characteristic curve (AUC) for VF was 0.448 (95\% confidence interval $(\mathrm{Cl})$ 0.274-0.622) for lead II of the CoECG, and 0.797 (95\%Cl 0.684-0.910) for lead II of the Radarcirc $^{\mathrm{TM}}$. The AUC for VF was $0.422(95 \% \mathrm{Cl} 0.219-0.626)$ for lead I of the CoECG, and $0.987(95 \% \mathrm{Cl} 0.975-$ 1.00) for lead I of the Radarcirc ${ }^{\mathrm{TM}}$.
\end{abstract}

Conclusions: Diagnoses based on the data from Radarcirc ${ }^{\mathrm{TM}}$ were more accurate in predicting rhythm during chest compressions than those based on data from the CoECG. (Circ J 2010; 74: 1339-1345)

Key Words: Cardiopulmonary resuscitation; Chest compressions; ECG; Ventricular fibrillation

$\mathbf{T}$ he importance of external chest compressions during cardiopulmonary resuscitation (CPR) was more strongly emphasized at the International Consensus Conference on Cardiopulmonary Resuscitation and Emergency Cardiovascular Care Science, and in the 2005 American Heart Association (AHA) guidelines for CPR and emergency cardiovascular care, than in preceding guidelines. ${ }^{1-8}$ However, during a cardiac rhythm check, chest compressions must be discontinued in order to avoid electrocardiograph (ECG) artifacts. ${ }^{9-14}$

A new multifunctional ECG, Radarcirc ${ }^{\mathrm{TM}}$ (Dainippon Sumitomo Pharma Co, Ltd, Osaka, Japan), has been developed for use in clinical settings that are subject to sudden impacts and vibrations, which, together with movement of the patient's body, can influence the accuracy of ECG output. Radarcirc $^{\mathrm{TM}}$ uses the Fluclet analytical system to enable accurate rhythm assessment and to reduce movement inter- ference. ${ }^{15,16}$

Although Radarcirc ${ }^{\mathrm{TM}}$ has been used in various emergency settings, including those where patients are transported in motor vehicles and where clinical tests are performed, the influence of artifacts generated by CPR (particularly those generated by chest compressions) has not been assessed for this device.

In the present study, we compared the performance of the Radacirc $^{\mathrm{TM}}$ multifunctional ECG with that of a conventional ECG (CoECG) in the CPR setting.

\section{Patient Population}

\section{Methods}

We studied ECG data from a total of 41 patients with cardiopulmonary arrest who were directly transported to The Trauma and Critical Care Center of Teikyo University Hospi-

Received November 30, 2009; revised manuscript received March 7, 2010; accepted March 9, 2010; released online May 27, 2010 Time for primary review: 23 days

Department of Emergency Medicine (G.S., N.M., T.S.), Trauma and Critical Care Center, Department of Medicine (Cardiology) (T.I.), Teikyo University School of Medicine, Tokyo; R\&D and Manufacturing Group (S.N.), Radarcirc Operations, Dainippon Sumitomo Pharma Co, Ltd, Osaka; and Anesthesiology and Critical Care Medicine (T.G.), Graduate School of Medicine, Yokohama City University, Yokohama, Japan

Names of grants.

Mailing address: Genji Shimpuku, MD, Department of Emergency Medicine, Trauma and Critical Care Center, Teikyo University School of Medicine, 2-11-1 Kaga, Itabashi-ku, Tokyo 173-8606, Japan. E-mail: pcprj589@yahoo.co.jp

ISSN-1346-9843 doi:10.1253/circj.CJ-09-0928

All rights are reserved to the Japanese Circulation Society. For permissions, please e-mail: cj@j-circ.or.jp 


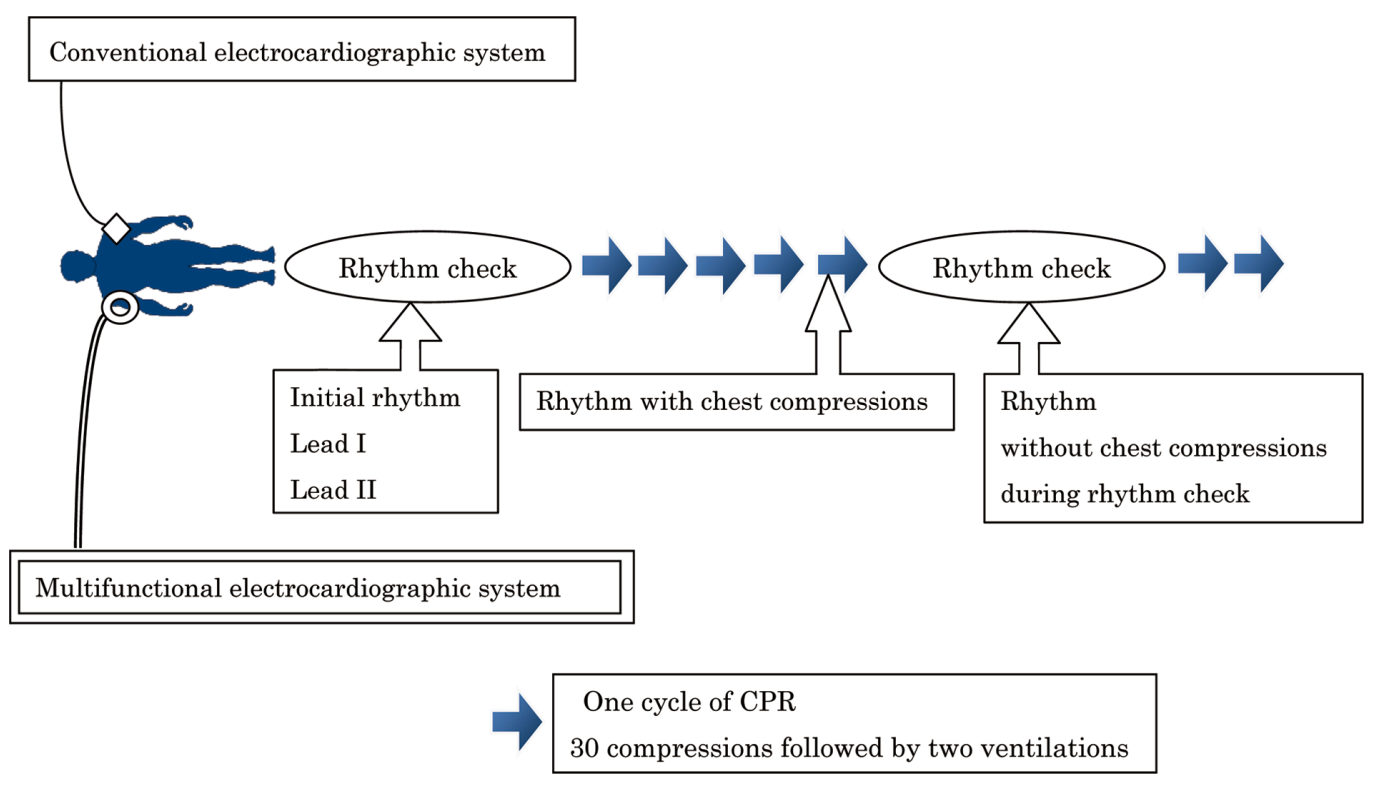

Figure 1. Schedule for measurements and assessment of rhythm types. On admission, during chest compression in the fifth cycle and just after the compression, we checked the cardiac rhythm and recorded these with the both monitoring systems. Lead I and II recordings were obtained using the Conventional electrocardiographic system and the Multifunctional electrocardiographic system. CPR, cardiopulmonary resuscitation.

tal School of Medicine, Tokyo, by ambulance between April and December 2007. The patient group comprised 27 males $(65.9 \%)$ and 14 females $(34.1 \%)$ with an average age of $70 \pm 12$ years (range $35-95$ years). We excluded patients with traumatic cardiopulmonary arrest, children under 12 years of age, and patients with do-not-attempt-resuscitation orders.

The protocol of a non-randomized, sequential self-controlled study was approved by the ethics board of Teikyo University Hospital. However, we could not explain the study's protocol to the patients before the study, because they were being transported by ambulance, and their relatives were very agitated.

\section{Protocol}

After patients had been transported to hospital by ambulance, they were administered CPR by the advanced cardiovascular life support (ACLS) team in accordance with the 2005 AHA guidelines. We checked the patients were in cardiopulmonary arrest not only by ECG, but also by checking for a carotid pulse.

We obtained ECG data using a CoECG and a multifunctional ECG simultaneously. The CoECG comprised a conventional ECG (Dynascope DS-7100 ${ }^{\mathrm{TM}}$; Fukuda Denshi Co, Ltd, Tokyo, Japan) and electrodes (Carbonrode II ${ }^{\mathrm{TM}}$; Fukuda Denshi Co, Ltd). The multifunctional ECG comprised a multifunctional ECG (Radarcirc ${ }^{\mathrm{TM}}$; Dainippon Sumitomo Pharma Co, Ltd) and a repositionable monitoring electrode (Red Dot ${ }^{\mathrm{TM}}$; 3M Health Care). For the multifunctional ECG system, disposable ECG electrode cover tapes (Echodrape $\mathrm{M}^{\mathrm{TM}}$; Fukuda Denshi Co, Ltd) were placed over the electrodes. The electrodes of the CoECG system were fastened to 3 areas of the patient's body (the right and left subclavicular areas, and the lowest left rib). Close by $(<5 \mathrm{~cm})$, the electrodes of the multifunctional ECG were fastened to 4 areas (the right and left subclavicular areas, and the lowest left and right ribs).
In accordance with the 2005 AHA guidelines, 1 cycle of CPR comprised 30 chest compressions and 2 ventilations. On admission, during chest compressions in the $5 \mathrm{~h}$ cycle and just after the compression, we checked the cardiac rhythm and recorded it using both ECG monitoring systems. Recording time was within approximately $10 \mathrm{~s}$, and did not disrupt CPR. Lead I and II recordings were obtained using the CoECG (CoI and CoII, respectively) and the multifunctional ECG (RI and RII, respectively) (Figure 1). When the cardiac rhythm changed during CPR, we measured it as another waveform.

Next, we measured the distance between the highest and lowest points of artifacts generated by chest compressions, using ECG tracings of the rhythm during chest compressions, to obtain the amplitude of the artifacts (Figure 2). Amplitudes are presented as median values.

After finishing all medical treatment and CPR, 5 cardiovascular specialists made their diagnoses on the basis of the ECG tracings. They worked as cardiologists in the hospital, but were separate from our department. They did not all check the rhythm at the same time nor could they make suggestions to our medical team. They were told whether chest compressions were being performed at the time of the ECG, but not which ECG was used to obtain the data.

Based on the ECG data, ventricular fibrillation (VF) was diagnosed if there were no normal QRS, P, and T waves, and a swinging baseline, with no carotid pulse; asystole was diagnosed if the baseline was flat, after checking the type of leads, the sensitivity of the monitors, and the lack of damage to the leads, with no carotid pulse. In this study, asystole included "P-wave asystole" in which persistent atrial activity exists without any ventricular response, and "agonal "or idioventricular electrical complexes that appear at a rate of less than $6 / \mathrm{min}$. The cardiologists diagnosed pulseless electrical activity (PEA) if there were several waves, other than VF 


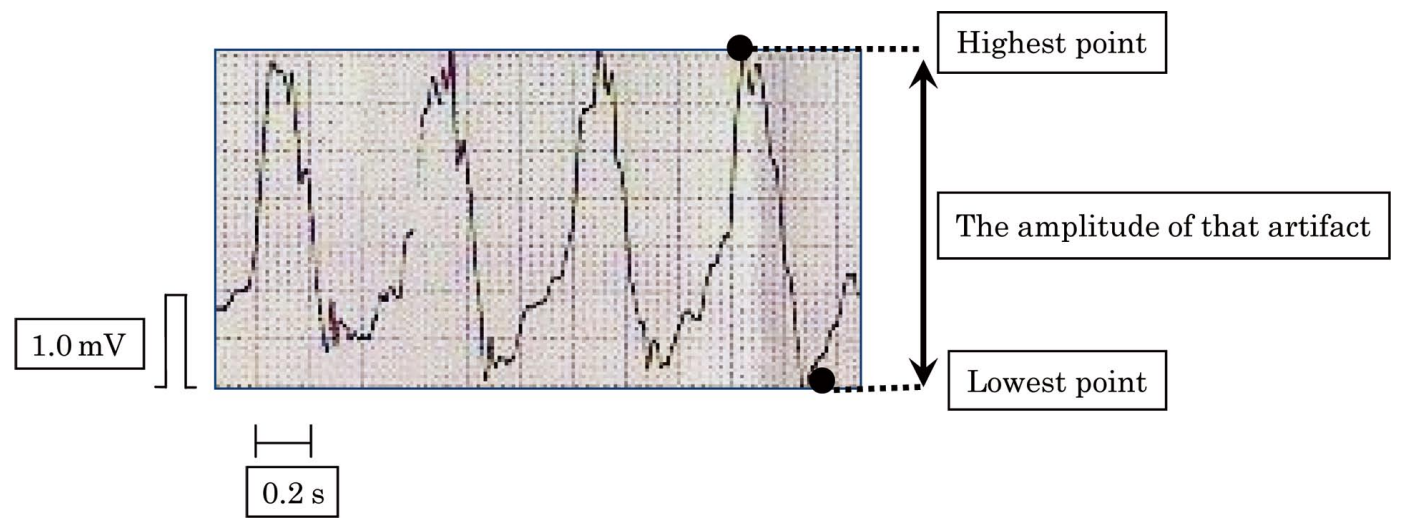

Figure 2. Definition and measurement of artifacts generated by chest compression. Each artifact was synchronized with chest compression. The distance between the highest and lowest points of an artifact was taken as the amplitude of that artifact.

\begin{tabular}{|c|c|c|c|c|}
\hline \multicolumn{5}{|c|}{ Coll without chest compressions } \\
\hline & Asystole & PEA & VF & Total \\
\hline \multicolumn{5}{|c|}{ Col with chest compressions } \\
\hline Asystole & 5 & 5 & 3 & 13 \\
\hline PEA & 25 & 18 & 8 & 51 \\
\hline VF & 2 & 4 & 0 & 6 \\
\hline Unclassified & 28 & 28 & 9 & 65 \\
\hline Total & 60 & 55 & 20 & 135 \\
\hline \multicolumn{5}{|c|}{ Coll with chest compressions } \\
\hline Asystole & 24 & 4 & 3 & 31 \\
\hline PEA & 28 & 37 & 9 & 74 \\
\hline VF & 5 & 4 & 0 & 9 \\
\hline Unclassified & 78 & 45 & 13 & 136 \\
\hline Total & 135 & 90 & 25 & 250 \\
\hline \multicolumn{5}{|c|}{ RI with chest compressions } \\
\hline Asystole & 107 & 3 & 0 & 110 \\
\hline PEA & 21 & 85 & 2 & 108 \\
\hline VF & 0 & 0 & 19 & 19 \\
\hline Unclassified & 7 & 2 & 4 & 13 \\
\hline Total & 135 & 90 & 25 & 250 \\
\hline \multicolumn{5}{|c|}{ RIl with chest compressions } \\
\hline Asystole & 62 & 4 & 0 & 66 \\
\hline PEA & 39 & 70 & 1 & 110 \\
\hline VF & 6 & 3 & 17 & 26 \\
\hline Unclassified & 28 & 13 & 7 & 48 \\
\hline Total & 135 & 90 & 25 & 250 \\
\hline
\end{tabular}

Coll, lead II of conventional electrocardiograph (ECG); PEA, pulseless electrical activity; VF, ventricular fibrillation; Col, lead I of conventional ECG; RI, lead I of multifunctional ECG; RII, lead II of multifunctional ECG.

The 5 cardiovascular specialists analyzed 1 piece of ECG data each, and a total of 250 Coll, RI and RII data and 135 Col data sets each. The diagnosis with each lead was compared with Coll without chest compressions.

and asystole, and no carotid pulse. PEA sometimes includes normal activity on the ECG monitor, but no carotid pulse. ${ }^{17}$ If a diagnosis could not be made because of artifacts from the chest compressions, the ECG data was regarded as 'unclassified'.

\section{Endpoints}

As the primary endpoint, we compared the accuracy of the diagnoses based on the RII and CoII data during chest compressions, relative to the diagnoses based on the CoII ECG data without chest compressions.

As a secondary endpoint, we compared the accuracy of diagnoses based on the RI and CoI data, and compared the amplitude of the artifacts in the RI and RII data with those in the CoII and CoI data (with chest compressions in all cases). 


\begin{tabular}{|c|c|c|c|c|c|c|c|}
\hline Lead & $\mathbf{n}$ & Sensitivity & Specificity & AUC & PPV & NPV & $\begin{array}{l}\text { Average of numbers } \\
\text { of cardiologists } \\
\text { made true diagnosis }\end{array}$ \\
\hline \multicolumn{8}{|l|}{ VF } \\
\hline Col & 135 & 0 & 0.95 & $0.422[0.219,0.626]$ & 0 & 0.85 & 0 \\
\hline Coll & 250 & 0 & 0.96 & $0.448[0.274,0.622]$ & 0 & 0.90 & 0 \\
\hline $\mathrm{RI}$ & 250 & 0.76 & 1.0 & $0.987[0.975,1.00]$ & 1.0 & 0.97 & 3.8 \\
\hline RII & 250 & 0.68 & 0.86 & $0.797[0.684,0.910]$ & 0.65 & 0.96 & 3.4 \\
\hline \multicolumn{8}{|l|}{ PEA } \\
\hline Col & 135 & 0.33 & 0.59 & $0.456[0.356,0.556]$ & 0.41 & 0.77 & 1.6 \\
\hline Coll & 250 & 0.41 & 0.77 & $0.599[0.521,0.678]$ & 0.50 & 0.70 & 2.1 \\
\hline RI & 250 & 0.94 & 0.86 & $0.880[0.831,0.929]$ & 0.79 & 0.97 & 4.7 \\
\hline RII & 250 & 0.78 & 0.75 & $0.743[0.679,0.807]$ & 0.64 & 0.86 & 3.9 \\
\hline \multicolumn{8}{|c|}{ Asystole } \\
\hline Col & 135 & 0.08 & 0.89 & $0.456[0.356,0.556]$ & 0.39 & 0.55 & 0.4 \\
\hline Coll & 250 & 0.18 & 0.94 & $0.599[0.521,0.678]$ & 0.77 & 0.49 & 0.9 \\
\hline RI & 250 & 0.79 & 0.97 & $0.889[0.846,0.933]$ & 0.97 & 0.80 & 4.0 \\
\hline RII & 250 & 0.46 & 0.97 & $0.743[0.679,0.807]$ & 0.94 & 0.60 & 2.3 \\
\hline
\end{tabular}

The overall predictive accuracy for all types of waveforms was $24.4 \%$ for Coll, $59.2 \%$ for RII, $17.0 \%$ for Col, and $84.8 \%$ for RI.

AUC, area under the receiver operating characteristic curve [95\% confidence interval]; PPV, positive predictive value; NPV, negative predictive value. Other abbreviations see in Table 1.

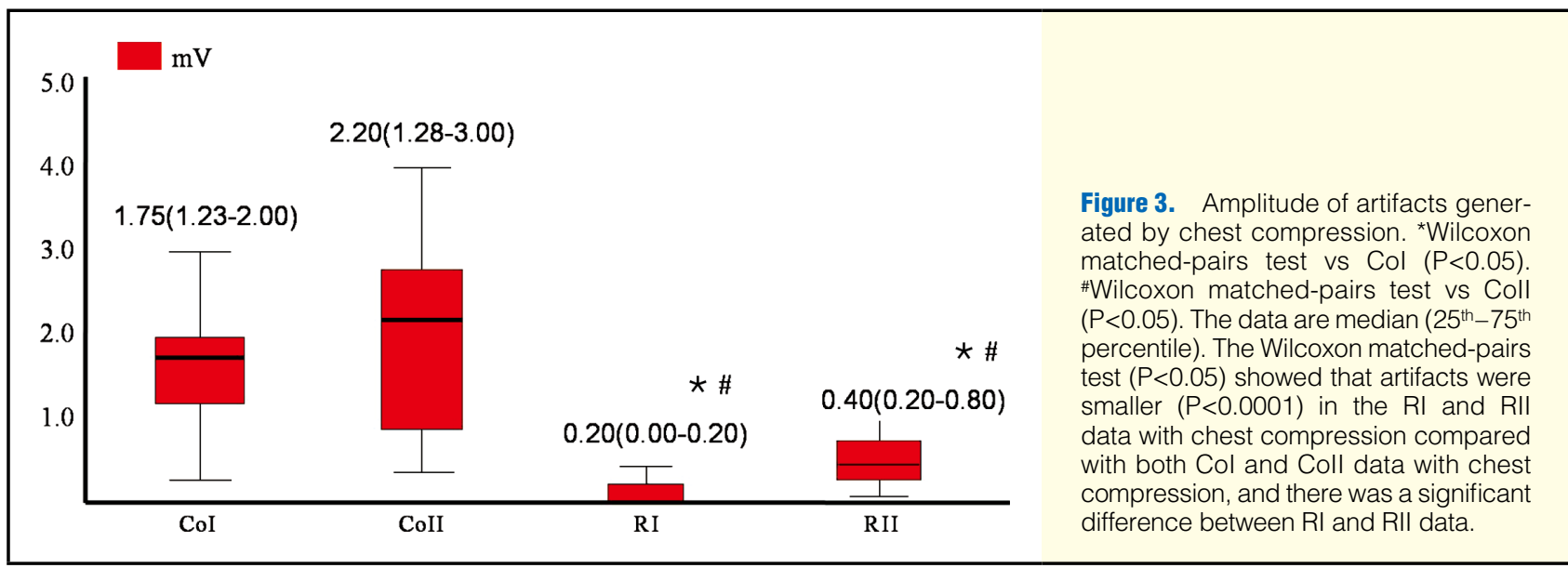

\section{Statistical Analysis}

Five diagnoses were obtained for each ECG data set, because the 5 cardiologists diagnosed individually. When the diagnosis during chest compressions was same as the diagnosis with CoII without chest compression, we judged that the diagnosis was true and that it could be made during chest compressions without artifacts. We judged "unclassified" as untrue. Diagnostic accuracy during chest compression consisted of several parts. For asystole, PEA, and VF waveforms, we calculated the sensitivity, specificity, positive predictive value (PPV), and negative predictive value (NPV). We averaged the numbers for the cardiologists who made a true diagnosis. The highest value was 5 and the lowest was 0 . The ability to make the same diagnosis as with CoII without chest compression was calculated by measuring the area under the receiver-operating characteristic curve (AUC). The Wilcoxon matchedpairs test was used to assess the significance of differences in artifact amplitude, with significance set at $\mathrm{P}<0.05$.

\section{Results}

We obtained 50 ECG tracings in CoII, RI, and RII from 41 patients, because 9 ECGs of 9 patients changed during CPR. Of these, there were 27 cases of asystole, 18 of PEA and 5 of VF diagnosed from the CoII data without chest compression. We obtained $27 \mathrm{ECG}$ tracings in $\mathrm{CoI}$ during chest compressions, because ECG data of 14 patients could not be recorded at the same time, or CPR had priority over the recording. Of these, there were 12 cases of asystole, 11 of PEA, and 4 of VF. None of the CoI ECGs changed during CPR. In this study, the ACLS team performed high-quality CPR without incident (ie, interrupted CPR or disconnected leads). ${ }^{18-21}$

Assessing these ECG tracings individually, the 5 cardiovascular specialists analyzed a total of 250 CoII, RI and RII data and 135 CoI data sets each (Table 1).

The overall predictive accuracy for all types of waveforms was $24.4 \%$ for CoII, and $59.2 \%$ for RII. In CoII for $\mathrm{VF}$, the sensitivity was 0 , specificity was 0.96 , PPV was 0 , 


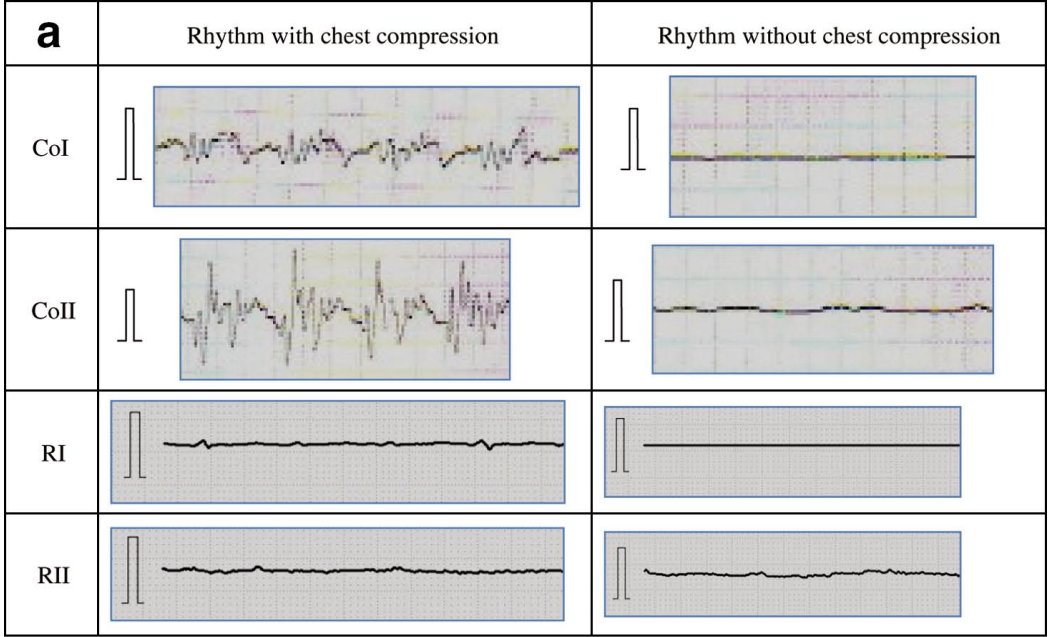

\begin{tabular}{|l|l|l|l|}
\hline b & Rhythm with chest compression & Rhythm without chest compression \\
\hline CoI & CoII & RI & RII
\end{tabular}

\begin{tabular}{|c|c|c|}
\hline C & Rhythm with chest compression & Rhythm without chest compression \\
\hline CoI & & \\
\hline CoII & & \\
\hline RI & & \\
\hline RII & & \\
\hline
\end{tabular}

Figure 4. Typical rhythm tracings. (a) 64-year-old woman. In both the RI and RII tracings, the waveform of the rhythm with chest compressions was consistent with that of the rhythm with no chest compressions. In this case, a diagnosis of asystole was possible during chest compressions. (b) 65-year-old man. In both the RI and RII tracings, the waveform of the rhythm with chest compressions was consistent with that of the rhythm with no chest compressions. In this case, a diagnosis of pulseless electrical activity was possible during chest compressions with no carotid pulse clinically. (c) 68-year-old man. In the RI and RII tracings, the waveform of the rhythm with chest compression was consistent with that of the rhythm without chest compression. In this case, a diagnosis of ventricular fibrillation was possible during chest compression.
NPV was 0.90, and the AUC was 0.448 (95\% confidence interval (CI) 0.274-0.622) (Table 2). In RII for VF, the sensitivity was 0.68 , specificity was 0.86 , PPV was 0.65 , NPV was 0.96, and the AUC was 0.797 (95\%CI 0.684-0.910). In CoII for PEA, the sensitivity was 0.41 , specificity was 0.77 , PPV was 0.50, NPV was 0.70, and the AUC was 0.599 (95\%CI 0.521-0.678). In RII for PEA, the sensitivity was
0.78, specificity was 0.75 , PPV was 0.64 , NPV was 0.86 , and the AUC was 0.743 (95\%CI 0.679-0.807). In CoII for asystole, the sensitivity was 0.18 , specificity was 0.94 , PPV was 0.77 , NPV was 0.49 , and the AUC was $0.634(95 \% \mathrm{CI}$ $0.535-0.732$ ). In RII for asystole, the sensitivity was 0.46 , specificity was 0.97 , PPV was 0.94 , NPV was 0.60 , and the AUC was 0.769 (95\%CI 0.709-0.829). 
The overall predictive accuracy for all types of waveforms was $17.0 \%$ for CoI, and $84.8 \%$ for RI. In CoI for VF, the sensitivity was 0 , specificity was 0.95 , PPV was 0 , NPV was 0.85 , and the AUC was 0.422 (95\%CI 0.219-0.626). In RI for VF, the sensitivity was 0.76 , specificity was 1.0 , PPV was 1.0, NPV was 0.97, and the AUC was $0.987(95 \% \mathrm{CI}$ 0.975-1.00). In CoI for PEA, the sensitivity was 0.33 , specificity was 0.59 , PPV was 0.41 , NPV was 0.77 , and the AUC was 0.456 (95\%CI 0.356-0.556). In RI for PEA, the sensitivity was 0.94 , specificity was 0.86 , PPV was 0.79 , NPV was 0.97 , and the AUC was 0.880 (95\%CI 0.831-0.929).

The amplitudes of the artifacts were as follows: $1.75(1.23-$ 2.00) $\mathrm{mV}$ for CoI, 2.20 (1.28-3.00) $\mathrm{mV}$ for CoII, $0.20(0.00$ $0.20) \mathrm{mV}$ for RI, and $0.40(0.20-0.80) \mathrm{mV}$ for RII. The Wilcoxon matched-pairs test $(\mathrm{P}<0.05)$ showed that artifacts were significantly smaller $(\mathrm{P}<0.0001)$ in the RI and RII data with chest compression compared with both $\mathrm{CoI}$ and CoII data with chest compression, and there was a significant difference between the RI and RII data (Figure 3).

The average number of cardiologists making a true diagnosis for VF was 0 for CoI, 0 for CoII, 3.8 for RI, and 3.4 for RII; for PEA it was 1.6 for CoI, 2.1 for CoII, 4.7 for RI, and 3.9 for RII; and for asystole it was 0.4 for CoI, 0.9 for CoII, 4.0 for RI, and 2.3 for RII.

Figure 4 shows representative cardiac rhythm tracings. In both the RI and RII tracings from a 64-year-old woman, the waveform of the rhythm with chest compressions was consistent with that of the rhythm with no chest compression, so in this case, a diagnosis of asystole was possible during chest compressions (Figure 4a). In both RI and RII tracings from a 65-year-old man, the waveform of the rhythm with chest compressions was consistent with that of the rhythm with no chest compression, so a diagnosis of PEA was possible during chest compressions with no carotid pulse clinically (Figure 4b). In both the RI and RII tracings obtained from a 68-year-old man, the waveform of the rhythm with chest compressions was consistent with that of the rhythm without chest compressions, so in this case, a diagnosis of VF was possible during chest compressions (Figure 4c).

\section{Discussion}

In the present study, tracings from a multifunctional ECG had significantly $(\mathrm{P}<0.0001)$ smaller artifacts during chest compressions relative to the CoECG. We propose some factors responsible for this reduction in artifacts.

The first factor the use of the Fluclet analytical system in the multifunctional ECG. ${ }^{15,16}$ The Fluclet software was developed with a focus on accurate real-time detection of the P, Q, R, S, and T peaks that characterize ECGs. ${ }^{15}$ The software uses a detection principle known as the "sandwich method", which is based on the ostensibly disadvantageous feature of the infinite impulse response (IIR) digital filter whereby the response is delayed relative to the original signal. The IIR digital filter is typically used to smooth out ECG waves that contain artifacts. Two waves generated by 2 IIR digital filters with different time delays are superimposed onto the original wave, in order to find a domain where both waves generated by the digital filters ascend. The highest value in the original wave within the identified domain is then recognized as the peak of a particular ECG wave. The negative peak of a particular ECG wave can be detected by finding the domain where both of the superimposed waves output by the digital filters descend and designating the lowest value in the original wave as the negative peak. This method enables accurate detection of peaks of particular ECG waves, even when baseline drifts are caused by impacts and vibrations, or when loud noises have occurred during recording. Because the ECG contains different frequency band components in 1 pulse, the peaks of particular ECG waves can be accurately detected by preparing the outputs for each component using IIR digital filters corresponding to each frequency band.

The second factor relates to lead choice. In the present study, artifacts were significantly less prevalent for lead I than lead II with both types of ECG. This is likely to be because the vector direction of measurement for lead I is not overly impinged upon by chest compression. With respect to diagnostic performance during chest compressions, lead I performed better for the multifunctional ECG, whereas lead II performed better for the CoECG. The reason for this result is not clear; however, it seems that diagnostic accuracy during chest compressions can be maximized by analyzing leads I and II in an integrated manner.

The overall predictive accuracy for all types of waveforms was $84.4 \%$ for RI, and $59.2 \%$ for RII. The predictive accuracies for VF were $76.0 \%$ for RI and $68.0 \%$ for RII; for PEA they were $94.4 \%$ for RI and $77.8 \%$ for RII; and for asystole they were $79.3 \%$ for RI and $45.9 \%$ for RII. The predictive accuracy for each waveform for RI was higher than for RII, because of the lower amplitude of the artifacts for RI. Comparing each patient, the amplitudes of the artifacts were very different from each other, because of manual chest compression. Comparing each waveform, the predictive accuracy for PEA was higher than for VF and asystole, for both RI and RII. The diagnosis of PEA might be easy, because PEA has several waves that are more similar to normal waves than do VF and asystole. When the waveform looked like asystole, the small artifact was diagnosed as PEA by some cardiologists. When the waveform looked like VF, the cardiologists diagnosed it as unclassified. This misdiagnosis for RI was smaller than for RII. Using RI for PEA is recommended rather than RII.

Gilmore et al proposed a model of cardiopulmonary arrest with VF that comprises 3 phases: the electrical, circulatory, and metabolic phases. ${ }^{22}$ Ideally, defibrillation should be instituted as soon as possible, in the electrical phase of VF. If the cardiac rhythm indicates that the arrest is in the electrical phase, and if a diagnosis of VF is possible using a multifunctional ECG during chest compression, the decision to perform defibrillation can be made early, and the defibrillator can be charged while chest compressions are performed, reducing the time to defibrillation. During the circulatory phase, tissue oxygenation is prioritized in order to achieve successful defibrillation. If the arrest is shown to be in the circulatory phase, chest compressions can be continued with little or no interruption, which may be beneficial for tissue oxygenation and may improve the resuscitation rate. If it becomes possible to distinguish between the electrical and circulatory phases, diagnoses will be able to be made during chest compression and appropriate treatment strategies selected according to the waveform detected.

\section{Study Limitations}

Only 135 CoI data sets were obtained from 27 ECGs, because CPR was prioritized over the recording. This is a limitation of clinical field studies. Another limitation is the small number of cases, especially VF. To succeed in resuscitation by defibrillation, quickly detecting patients in the electrical phase of VF is very useful with chest compressions. The 
second limitation is the way that the artifacts were created. In accordance with the 2005 AHA guidelines manual chest compression was performed, but this causes the artifact of each wave to be different. It may be better to use mechanical CPR in the hospital setting. The third limitation is the cover tapes. In the present study, electrode cover tapes were used with the multifunctional ECG electrodes, but not with the CoECG electrodes, in order to affix the electrodes firmly to the patient's body. The electrodes of the multifunctional ECG system were fastened to 4 areas of the body. In the emergency setting this would take more time than for the CoECG. The difference between lead I and lead II must be studied in future research.

Although future research and development in the field of multifunctional ECGs is expected to contribute to further reduction in ECG artifacts, more research is needed on identifying the electrical, circulatory, and metabolic phases of VF. It is also anticipated that this research will find application in the waveform analysis devices of automated external defibrillators.

\section{Conclusion}

Diagnoses based on the data from a new multifunctional ECG were more accurate in predicting rhythm during chest compressions than those based on the data from a CoECG. The multifunctional ECG had significantly smaller artifacts during chest compressions relative to the CoECG.

\section{Disclosure}

Shinya Nagata is an employee of Dainippon Sumitomo Pharma Co, Ltd. Dainippon Sumitomo Pharma paid for the writing and translation assistance. No other authors have conflicts of interest to declare.

\section{References}

1. International Liaison Committee on Resuscitation. 2005 International consensus on cardiopulmonary resuscitation and emergency cardiovascular care with treatment recommendations. Circulation 2005; 112: IIII-1 - III-136.

2. Eftestol T, Sunde K, Steen PA. Effects of interrupting precordial compressions on the calculated probability of defibrillation success during out-of-hospital cardiac arrest. Circulation 2002; 105: 2270 2273 .

3. Yu T, Weil MH, Tang W, Sun S, Klouche K, Povoas H, et al. Adverse outcomes of interrupted precordial compressions during automated defibrillation. Circulation 2002; 106: 368-372.

4. Sayre MR, Berg RA, Cave DM, Page RL, Potts J, White RD. Handsonly (compression-only) cardiopulmonary resuscitation: A call to action for bystander response to adults who experience out-of-hospital sudden cardiac arrest. Circulation 2008; 117: 2162-2167.

5. Jacobs I, Nadkarni V, Bahr J, Berg RA, Billi JE, Bossaert L, et al; International Liason Committee on Resusitation. Cardiac arrest and cardiopulmonary resuscitation outcome reports: Update and simplification of the Utstein templates for resuscitation registries: A statement for healthcare professionals from a task force of the international liaison committee on resuscitation (American Heart Asso- ciation, European Resuscitation Council, Australian Resuscitation Council, New Zealand Resuscitation Council, Heart and Stroke Foundation of Canada, InterAmerican Heart Foundation, Resuscitation Council of Southern Africa). Resuscitation 2004; 63: 233-249.

6. Kern KB, Hilwig RW, Berg RA, Sanders AB, Ewy GA. Importance of continuous chest compressions during pulmonary resuscitation: Improved outcome during a simulated single lay-rescuer scenario. Circulation 2002; 105: 645-649.

7. Berg RA, Hilwig RW, Kern KB, Ewy GA. "Bystander" chest compressions and assisted ventilation independently improve outcome from piglet asphyxial pulseless "cardiac arrest". Circulation 2000; 101: $1743-1748$.

8. Berg RA, Kern KB, Hilwig RW, Ewy GA. Assisted ventilation during 'bystander' CPR in a swine acute myocardial infarction model does not improved outcome. Circulation 1997; 96: 4364-4371.

9. Guidelines for cardiopulmonary resuscitation and emergency cardiovascular care, part 4: The automated external defibrillator. Circulation 2000; 102(Suppl I): I-60-I-76.

10. Berger RD, Palazzolo J, Halperin H. Rhythm discrimination during uninterrupted CPR using motion artifact reduction system. Resuscitation 2007; 75: $145-152$.

11. Aramendi E, de Gauna SR, Irusta U, Ruiz J, Arcocha MF, Ormaetxe JM. Detection of ventricular fibrillation in the presence of cardiopulmonary resuscitation artefacts. Resuscitation 2007; 72: 115-123.

12. Eilevstjonn J, Eftestol T, Aase SO, Myklebust H, Husoy JH, Steen PA. Feasibility of shock advice analysis during CPR through removal of CPR artefacts from the human ECG. Resuscitation 2004; 61: $131-141$.

13. Aase SO, Eftesol T, Husoy JH, Sunde K, Steen PA. CPR artifact removal from human ECG using optimal multichannel filtering. IEEE Trans Biomed Eng 2000; 47: 1440-1449.

14. Berg RA, Hilwig RW, Kern KB, Sanders AB, Xavier LC, Ewy GA. Automated external defibrillation versus manual defibrillation for prolonged ventricular fibrillation: Lethal delays of chest compressions before and after countershocks. Ann Emerg Med 2003; 42: $458-467$.

15. Nagai R, Nagata S. New algorithms for real-time, $24 \mathrm{hr}$ continuous and noise-adjusted power spectral analysis of heart rate and blood pressure fluctuations in conscious rats. J Pharmacol 1996; 72: 355364.

16. Nagai R, Nagata S. New algorithmic-based digital filter processing system for real-time continuous blood pressure measurement and analysis in conscious rats. Comput Biol Med 1995; 25: 483-494.

17. American Heart Association. 2005 guidelines for cardiopulmonary resuscitation and emergency cardiovascular care. Circulation 2005; 112: $58-66$.

18. Nagao K, Kikushima K, Watanabe K, Tachibana E, Tominaga Y, Tada K, et al. Early induction of hypothermia during cardiac arrest improves neurological outcomes in patients with out-of-hospital cardiac arrest who undergo emergency cardiopulmonary bypass and percutaneous coronary intervention. Circ J 2010; 74: 77-85.

19. Aoyama N, Imai H, Kono K, Kato S, Fukuda N, Kurosawa T, et al. Patient selection and therapeutic strategy for emergency percutaneous cardiopulmonary system in cardiopulmonary arrest patients. Circ J 2009; 73: 1416-1422.

20. SOS-KANTO study group. Comparison of arterial blood gases of laryngeal mask airway and bag-valve-mask ventilation in out-ofhospital cardiac arrests. Circ J 2009; 73: 490-496.

21. SOS-KANTO Committee. Incidence of ventricular fibrillation in patients with out-of-hospital cardiac arrest in Japan: Survey of survivors after out-of-hospital cardiac arrest in Kanto area (SOS-KANTO). Circ J 2005; 69: 1157-1162.

22. Gilmore CM, Rea TD, Becker LJ, Eisenberg MS. Three-phase model of cardiac arrest: Time-dependent benefit of bystander cardiopulmonary resuscitation. Am J Cardiol 2006; 98: 497-499. 